Supporting information for

\title{
On-fly femtosecond-laser fabrication of self-organized plasmonic nanotextures for chemo- and biosensing applications
}

\author{
Aleksandr Kuchmizhak, ${ }^{a, b, *}$ Evgenii Pustovalov, ${ }^{a}$ Sergey Syubaev, ${ }^{a, b}$ Oleg Vitrik, ${ }^{\text {a,b }}$ Yuri \\ Kulchin, ${ }^{b}$ Aleksey Porfirev, ${ }^{b, c}$ Svetlana Khonina, ${ }^{c}$ Sergey Kudryashov, ${ }^{\text {b,de }}$ Pavel Danilov, \\ Andrey Ionin ${ }^{\mathrm{e}}$ \\ ${ }^{a}$ School of Natural Sciences, Far Eastern Federal University (FEFU), 8 Sukhanova str., Vladivostok 690041, Russia \\ ${ }^{\mathrm{b}}$ Institute of Automation and Control Processes (IACP), Far Eastern Branch of Russian Academy of Science (FEB RAS), 5 Radio \\ Str., Vladivostok 690041, Russia \\ ${ }^{\mathrm{c}}$ Samara State Aerospace University, 34 Moskovskoye shosse, Samara 443086, Russia \\ ITMO University, Kronverkskiy prospect 49, St. Petersburg 197101, Russia. \\ ${ }^{\text {e}}$ Lebedev Physical Institute, Leninskiy prospect 53, Moscow 119991, Russia. \\ *alex.iacp.dvo@mail.ru
}




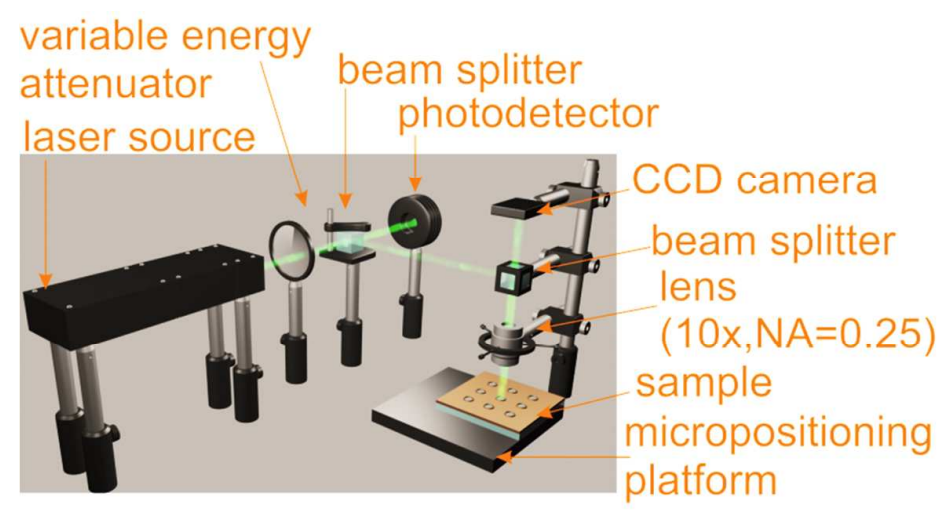

Figure S1. Schematic of the experimental setup for laser nanotexturing of thick metal films on the glass substrates.

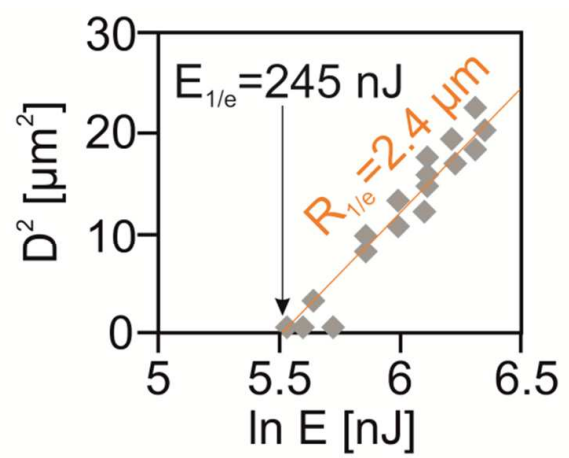

Figure S2. Dependence of the squared diameter $\mathrm{D}^{2}$ of the spallative crater on the incident pulse energy E.

Supplementary note to Figure S2. According to ${ }^{1}$, the angular slope (orange line) corresponds to the squared 1/e-diameter of the laser energy deposition, exhibiting the $\mathrm{R}_{1 / \mathrm{e}}$-radius of $2.4 \mu \mathrm{m}$, while the intersection of this fitting line with the horizontal axis defines the threshold energy, required for formation of the spallative crater, of $E=245 \mathrm{~nJ}$ (the corresponding peak fluence $\sim 1.4 \mathrm{~J} / \mathrm{cm}^{2}$ ).
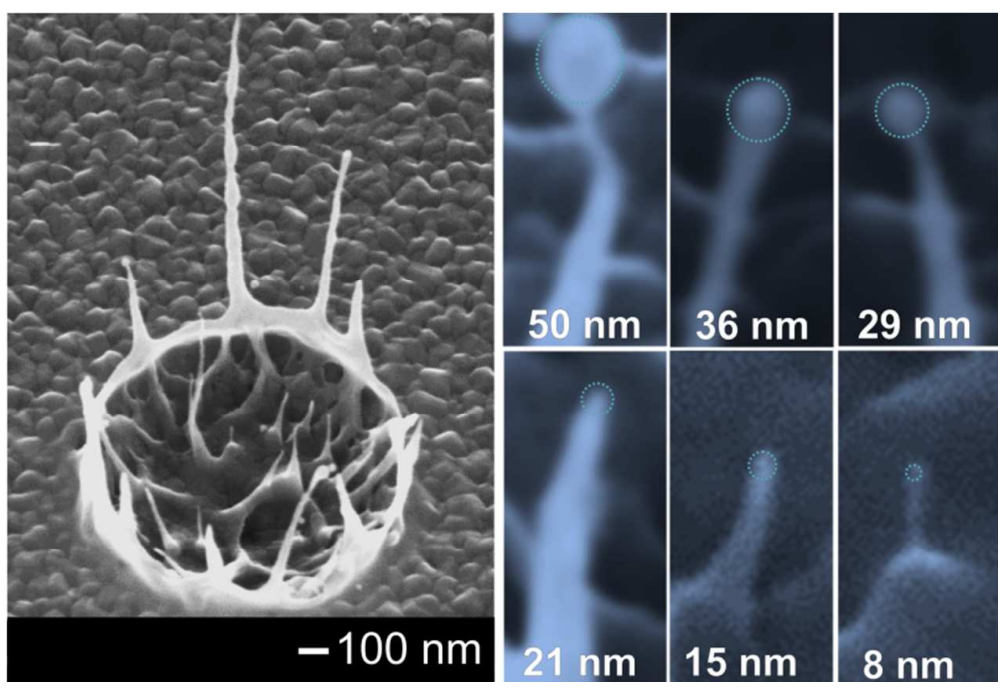

Figure S3. High-resolution SEM images (view angle of $45^{\circ}$ ), visualizing separate nanotips inside the spallative crater and their characteristic dimensions. 


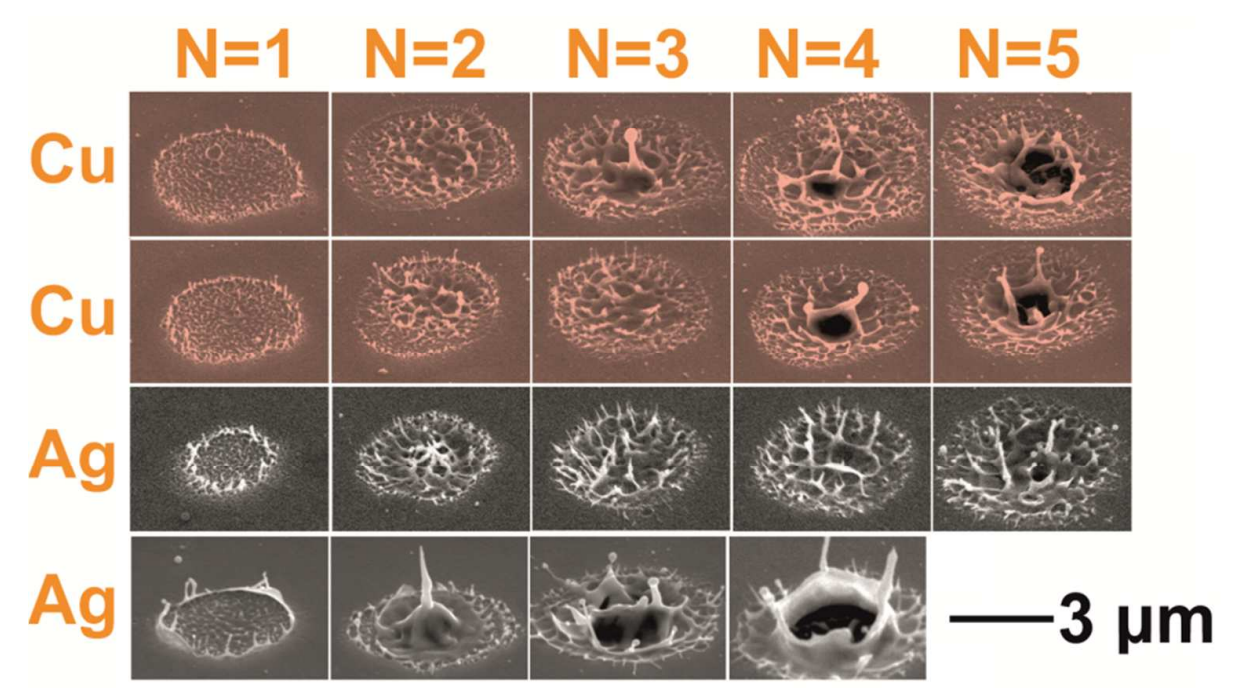

Figure S4. Sets of SEM images (view angle of $45^{\circ}$ ), illustrating different scenarios of the surface nanotopography evolution versus the number of applied laser pulses for the $\mathrm{Cu}$ and $\mathrm{Ag}$ films at the peak fluences of 1.3 (two upper-most rows), 1.85 (third row) and $2.05 \mathrm{~J} / \mathrm{cm}^{2}$ (forth row), respectively.



Figure S5. Normalized SEPL spectra measured from the R6G layer covering the single- (green curve, $\mathrm{N}=1$ ), double- (orange curves) and triple-pulse (black curves) spallative craters under the $\mathrm{s}$ - and p-polarized lateral exciting illumination. Each SEPL spectrum was normalized on the spectrum measured from the R6G layer, covering a non-ablated (smooth) Ag film region of the same size. The inset shows the reference side-view SEM image (view angle of $45^{\circ}$ ) of the corresponding spallative craters. The multi-pulse irradiation was performed at the constant peak fluence of $\sim 1.9 \mathrm{~J} / \mathrm{cm}^{2}$ with a few-second delay between the pulses. 


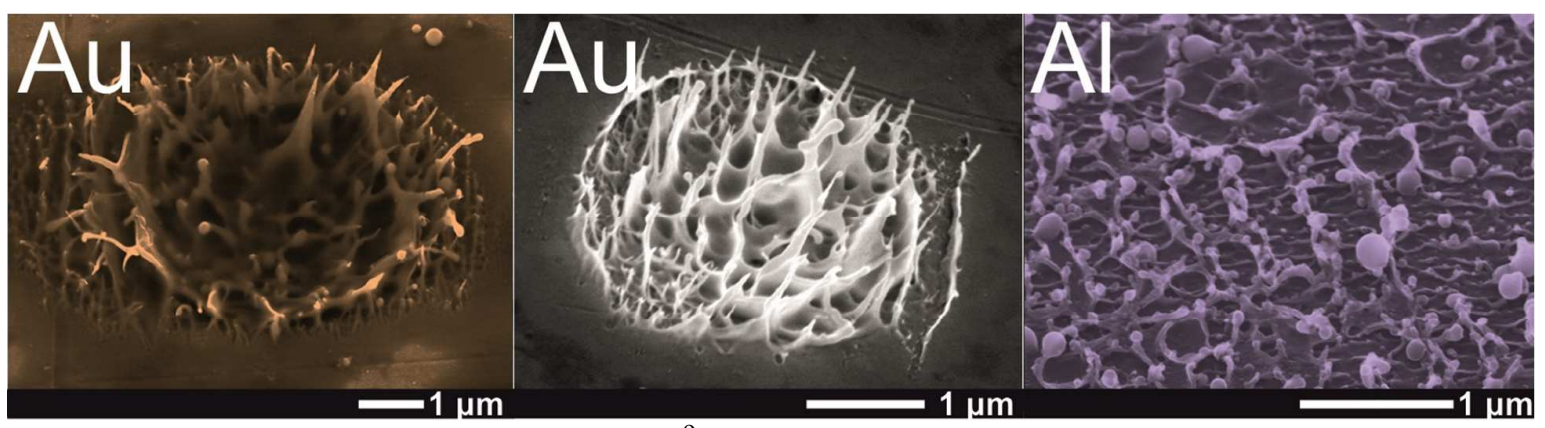

Figure S6. SEM images (view angle of $45^{\circ}$ ) of spallative craters produced on the surfaces of the 500-nm thick $\mathrm{Au}$ film (left), bulk Au (middle) and 500-nm thick Al film (right) under singlepulse irradiation.

\section{References}

(1) Liu, J. M. Simple Technique for Measurements of Pulsed Gaussian-Beam Spot Sizes. Opt. Lett. 1982, 7, 196-198. 